\title{
CHARACTERIZATIONS OF FUNCTIONS WITH HIGHER ORDER DERIVATIVES IN $\mathscr{L}_{p}$
}

\author{
BY \\ J. W. JEROME AND L. L. SCHUMAKER( $\left.{ }^{1}\right)$
}

1. Introduction. In $1910 \mathrm{~F}$. Riesz [3], [4] established the following characterization for absolutely continuous functions with first derivatives in $\mathscr{L}_{p}$ :

THEOREM 1.1 (F. RIESz). Let $(a, b)$ be a possibly infinite interval in $\boldsymbol{R}$. A necessary and sufficient condition that the function $F$ be the integral of an element of the space $\mathscr{L}_{p}(a, b), 1<p<\infty$, is that the sums

$$
\sum_{i=0}^{n-1} \frac{\left|F\left(x_{i+1}\right)-F\left(x_{i}\right)\right|^{p}}{\left(x_{i+1}-x_{i}\right)^{p-1}}
$$

formed for every finite system of points $a<x_{0}<x_{1}<\cdots<x_{n}<b$ have a finite least upper bound.

In 1964 Schoenberg [5] obtained an analogous characterization for the classes $\mathscr{H}_{2}^{m}$ for $1 \leqq m$, where in general

$$
\begin{aligned}
& \mathscr{H}_{p}^{m}(a, b)=\left\{f: f^{(m-1)} \in A C(a, b), f^{(m)} \in \mathscr{L}_{p}(a, b)\right\}, \\
& A C(a, b)=\{f: f \text { is abs. cont. on finite subintervals of }(a, b)\} .
\end{aligned}
$$

In particular, exploiting properties of natural spline functions, he obtained the following two results, where $\Delta_{h}^{m}$ denotes the $m$ th successive difference.

Theorem 1.2 (Schoenberg). Let $F \in C[a, b]$ with $-\infty<a<b<\infty$. Then $F \in$ $\mathscr{H}_{2}^{m}(a, b)$ if and only if there is a constant $K$ independent of $n$ such that on dividing $[a, b]$ into $n$ equal parts by means of the points $x_{i}=a+(b-a) i / n$ and setting $h=(b-a) / n$ we have

$$
h \sum_{i=0}^{n-m}\left(\frac{\Delta_{h}^{m} F\left(x_{i}\right)}{h^{m}}\right)^{2} \leqq K \text { for all } n
$$

Theorem 1.3 (Schoenderg). Let $F \in C(-\infty, \infty)$. Then $F \in \mathscr{H}_{2}^{m}(-\infty, \infty)$ if and only if there is a constant $K$ independent of $h$ such that

$$
h \sum_{i=-\infty}^{\infty}\left(\Delta_{h}^{m} F(i h) / h^{m}\right)^{2} \leqq K \text { for all } h>0
$$

Presented to the Society, August 29, 1968; received by the editors August 14, 1968 and, in revised form, February 3, 1969.

(1) Sponsored by the Mathematics Research Center, United States Army, Madison, Wisconsin, under Contract No. DA-31-124-ARO-D-462. 
We shall establish the following direct generalization of Riesz's theorem for arbitrary $1 \leqq m$ and $1<p<\infty$, where the symbol $\left[F\left(x_{i}\right), \ldots, F\left(x_{i+m}\right)\right]$ denotes the $m$ th divided difference of $F$ on the points $x_{i}<\cdots<x_{i+m}$.

THeOREM 1.4. Let $(a, b)$ be a possibly infinite interval in $\boldsymbol{R}$. Then $F \in \mathscr{H}_{p}^{m}(a, b)$ if and only if there exists a constant $K$ independent of $n$ such that

$$
\sum_{i=0}^{n-m}\left|\left[F\left(x_{i}\right), \ldots, F\left(x_{i+m}\right)\right]\right|^{p}\left(x_{i+m}-x_{i}\right) \leqq K
$$

for every choice of $n$ and

$$
a<x_{0}<x_{1}<\cdots<x_{n}<b .
$$

In particular, if $F \in \mathscr{H}_{p}^{m}$ then $K$ may be taken to be $\left(m /[(m-1) !]^{p}\right)\left\|F^{(m)}\right\|_{\mathscr{L}_{p}}^{p}$.

In this theorem, no assumption on the continuity of $F$, as is required in Theorems 1.2 and 1.3 , is necessary. Indeed, the random spacing of the division points eliminates the possibility that a discontinuous additive function can satisfy (1.2) for every partition of $(a, b)$. The proof of Theorem 1.4 will be carried out in $\S 3$, where we will also obtain intermediate results and characterizations subsuming Theorems 1.2 and 1.3. To this end we shall introduce in $\$ 2$ the notion of spline functions which solve appropriate minimization problems in $\mathscr{L}_{p}$. An additional characterization of $\mathscr{H}_{p}^{m}(a, b)$, in terms of these splines, will be given in the final theorem of $\S 3$ which summarizes the major results of the paper.

2. $p$-splines. Let $a \leqq x_{0}<x_{1}<\cdots<x_{n} \leqq b$ and $r=\left(r_{0}, r_{1}, \ldots, r_{n}\right) \in \boldsymbol{R}^{n+1}$ be prescribed. Consider the minimization problem

$$
\begin{aligned}
\left\|s^{(m)}\right\|_{\mathscr{L}_{p}} & =\min _{U(r)}\left\|f^{(m)}\right\|_{\mathscr{L}_{p},} \\
U(r) & =\left\{f \in \mathscr{H}_{p}^{m}: f\left(x_{i}\right)=r_{i}, 0 \leqq i \leqq n\right\} .
\end{aligned}
$$

$s$ will be called a $p$-spline associated with the partition $a \leqq x_{0}<x_{1}<\cdots<x_{n} \leqq b$, or, more simply, a $p$-spline if $s \in \mathscr{H}_{p}^{m}$ and $s$ satisfies (2.1) for some $r \in \boldsymbol{R}^{n+1}$. To show that (2.1) admits of a solution we begin by remarking that $\mathscr{H}_{p}^{m}$ is well known to be a Banach space under a variety of equivalent norms, e.g.

$$
\|f\|_{\mathscr{H}_{p}^{m}}=\sum_{i=1}^{m}\left|f\left(y_{i}\right)\right|+\left\|f^{(m)}\right\|_{\mathscr{L}_{p}}
$$

where $\left\{y_{i}\right\}_{1}^{m} \subset(a, b)$. Since convergence in $\mathscr{H}_{p}^{m}$ implies in particular uniform convergence on compact sets, it is easily seen that the flat $U(r)$ is closed in $\mathscr{H}_{p}^{m}$. Moreover, $(d / d x)^{m}$ is a bounded linear operator mapping $\mathscr{H}_{p}^{m}$ onto $\mathscr{L}_{p}$. But then the hypotheses of Lemma IV.2.9 of [1] are satisfied, and thus $(d / d x)^{m} U(r)$ is closed in $\mathscr{L}_{p}$. Since $(d / d x)^{m} U(r)$ is also convex and $\mathscr{L}_{p}$ is uniformly convex, we conclude that $(d / d x)^{m} U(r)$ possesses a unique element $g \in \mathscr{L}_{p}$ of minimal norm. Clearly there exists at least one $s \in U(r)$ such that $s^{(m)}=g$ and thus $s$ solves (2.1). In 
addition, it is immediate that $s$ is the unique solution of (2.1) if and only if $\pi_{m-1}$ $\cap U(0)=(0)$, where $\pi_{m-1}$ is the class of polynomials of degree at most $m-1$. In particular, $s$ is unique whenever $n \geqq m-1$.

$p$-splines associated with more general minimization problems (cf. [2] for $p=2$ ) can also be defined. Indeed, for general $m$ th order linear differential operators $L$ with continuous coefficients and nonvanishing leading coefficient, there exists a $p$-spline minimizing $\|L f\|_{\mathscr{L}_{p}}$ over the flat $U(r)=\left\{f \in \mathscr{H}_{p}^{m}: \lambda_{i} f=r_{i}, 0 \leqq i \leqq n\right\}$ defined by an arbitrary set of $n+1$ continuous linear functionals $\left\{\lambda_{i}\right\}_{0}^{n}$ such that $U(r) \neq \varnothing$.

General properties of $p$-splines will be studied elsewhere. For the present purposes we need only the following convergence result.

Lemma 2.1. For $i \geqq 1$ let $U_{i}$ be a flat defined by the mesh $\left\{x_{i, j}\right\}_{j=0}^{n_{i}}$ and the data

$$
r_{i}=\left(r_{i 0}, \ldots, r_{i n_{i}}\right)
$$

as in (2.1) with $n_{1} \geqq m-1$ and $U_{i+1} \subset U_{i}$. Let $s_{i}$ be the p-spline solving (2.1) for $U_{i}$. Then $\bigcap_{i=1}^{\infty} U_{i} \neq \varnothing$ if and only if there exists a constant $K$ such that $\left\|s_{i}^{(m)}\right\|_{\mathscr{L}_{p}} \leqq$ for all $i$. The constant may be taken as $\left\|f^{(m)}\right\|_{\mathscr{L}_{p}}$ for any $f \in \bigcap_{i=1}^{\infty} U_{i}$. Moreover, if $\left\|s_{i}^{(m)}\right\|_{\mathscr{L}_{p}} \leqq K$, then $s_{i}$ converges in the sense of $\mathscr{H}_{p}^{m}$ to a function $f \in \bigcap_{i=1}^{\infty} U_{i}$.

Proof. Suppose $f \in \bigcap_{i=1}^{\infty} U_{i}$. Then we infer immediately that $\left\|s_{i}^{(m)}\right\|_{\mathscr{L}_{p}} \leqq\left\|f^{(m)}\right\|_{\mathscr{L}_{p}}$. Conversely, suppose $\left\|s_{i}^{(m)}\right\|_{\mathscr{L}_{p}} \leqq K$. Since $U_{i+1} \subset U_{i}$ we have $\left\|s_{i}^{(m)}\right\|_{\mathscr{L}_{p}} \leqq\left\|s_{i+1}^{(m)}\right\|_{\mathscr{L}_{p}}$. It follows that $\left\|s_{i}^{(m)}\right\|_{\mathscr{L}_{p}}$ is a bounded monotone increasing sequence which converges to a limit $A$. Assume first that $A>0$. Then for arbitrary $0<\varepsilon<1$ we have for sufficiently large $i$ and all $j \geqq i$

$$
1-\varepsilon \leqq\left\|s_{i}^{(m)}\right\|_{\mathscr{L}_{p}} / A \leqq\left\|\left(s_{i}^{(m)}+s_{j}^{(m)}\right) / 2\right\|_{\mathscr{L}_{p}} / A
$$

since $\left(s_{i}+s_{j}\right) / 2 \in U_{i}$. But then by the uniform convexity of $\mathscr{L}_{p}$ for $1<p<\infty$ it follows that

$$
\left\|s_{j}^{(m)}-s_{i}^{(m)}\right\|_{\mathscr{L}_{p}} \leqq A \delta(\varepsilon)
$$

with $\delta(\varepsilon) \rightarrow 0$ as $\varepsilon \rightarrow 0$, i.e. the sequence $s_{i}^{(m)}$ is Cauchy in $\mathscr{L}_{p}$ and thus converges. Since all of the $s_{i} \in U_{1}$, i.e. they agree on the mesh $\left\{x_{1, j}\right\}_{0}^{n_{1}}$, it follows that the $s_{i}$ converge to a function $f \in \mathscr{H}_{p}^{m}$ which is easily seen to be in $\bigcap_{i=1}^{\infty} U_{i}$ and the proof is complete for $A>0$. On the other hand if $A=0$, i.e. $\left\|s_{i}^{(m)}\right\|_{\mathscr{L}_{p}}=0$ for all $i$, then $s_{i}^{(m)}$ is trivially Cauchy in $\mathscr{L}_{p}$ and hence $s_{i}$ converges in $\mathscr{H}_{p}^{m}$ as before.

The following characterization theorem is an easy consequence of Lemma 2.1.

TheOREM 2.2. Let $F \in C[a, b]$ and let $\Gamma_{i}=\left\{x_{i, j}\right\}_{j=0}^{n_{i}}$ be a sequence of meshes in $[a, b]$ with $n_{1} \geqq m-1, \Gamma_{i} \subset \Gamma_{i+1}$, and $\bigcup_{i=1}^{\infty} \Gamma_{i}$ is dense in $[a, b]$. Define the flats

$$
U_{i}=\left\{f \in \mathscr{H}_{p}^{m}: f\left(x_{i, j}\right)=F\left(x_{i, j}\right), 0 \leqq j \leqq n_{i}\right\}
$$

and let $s_{i}$ be the p-splines solving (2.1) with respect to $U_{i}$. Then $F \in \mathscr{H}_{p}^{m}$ if and only if there exists a constant $K$ independent of $i$ such that

$$
\left\|s_{i}^{(m)}\right\|_{\mathscr{L}_{p}} \leqq K \text {. }
$$

In particular, if $F \in \mathscr{H}_{p}^{m}$ then $K$ may be taken as $\left\|F^{(m)}\right\|_{\mathscr{L}_{p}}$. 
Proof. By the definition of the $U_{i}$ we have $U_{i+1} \subset U_{i}$. Now suppose $F \in \mathscr{H}_{p}^{m}$. Then clearly $F \in \bigcap_{i=1}^{\infty} U_{i}$ and thus by Lemma $2.1\left\|s_{i}^{(m)}\right\|_{\mathscr{L}_{p}} \leqq\left\|F^{(m)}\right\|_{\mathscr{L}_{p}}$. Conversely, if (2.2) is satisfied then Lemma 2.1 implies that $s_{i}$ converges to a function $f \in \bigcap_{i=1}^{\infty} U_{i}$. We claim $f \equiv F$. Indeed, $f(x)-F(x)=0$ for $x \in \bigcup_{i=1}^{\infty} \Gamma_{i}$ which is a dense set in $[a, b]$, and hence by the continuity of $f-F$ we conclude that $f(x)=F(x)$ for all $x \in[a, b]$, i.e. $F \in \mathscr{H}_{p}^{m}$.

3. Proof of Theorem 1.4. We begin by showing that $F \in \mathscr{H}_{p}^{m}$ implies that $F$ satisfies (1.2). We recall the well-known identity

where

$$
\left[F\left(x_{i}\right), \ldots, F\left(x_{i+m}\right)\right]=\frac{F^{(m-1)}\left(\xi_{i}\right)-F^{(m-1)}\left(\eta_{i}\right)}{(m-1) !\left(x_{i+m}-x_{i}\right)},
$$

$$
x_{i} \leqq \eta_{i}<x_{i+m-1} \text { and } x_{i+1}<\xi_{i} \leqq x_{i+m} .
$$

Substituting in (1.2) we obtain

$$
\begin{aligned}
I\left(x_{0}, \ldots, x_{n}\right) & \triangleq((m-1) !)^{p} \sum_{i=0}^{n-m}\left|\left[F\left(x_{i}\right), \ldots, F\left(x_{i+m}\right)\right]\right|^{p}\left(x_{i+m}-x_{i}\right) \\
& =\sum_{i=0}^{n-m} \frac{\left|F^{(m-1)}\left(\xi_{i}\right)-F^{(m-1)}\left(\eta_{i}\right)\right|^{p}}{\left(x_{i+m}-x_{i}\right)^{p-1}} \leqq \sum_{i=0}^{n-m} \frac{\left|F^{(m-1)}\left(\xi_{i}\right)-F^{(m-1)}\left(\eta_{i}\right)\right|^{p}}{\left|\xi_{i}-\eta_{i}\right|^{p-1}} \\
& \leqq \sum_{i=0}^{n-m} \frac{\left|\int_{\eta_{i}}^{\xi_{i}}\right| F^{(m)}(x)|d x|^{p}}{\left|\xi_{i}-\eta_{i}\right|^{p-1}} .
\end{aligned}
$$

Upon applying Hölder's inequality to the last expression we obtain

and by (3.1)

$$
I\left(x_{0}, \ldots, x_{n}\right) \leqq\left.\sum_{i=0}^{n-m}\left|\int_{n_{i}}^{\xi_{1}}\right| F^{(m)}(x)\right|^{p} d x \mid
$$

$$
I\left(x_{0}, \ldots, x_{n}\right) \leqq m\left\|F^{(m)}\right\|_{\mathscr{L}_{p}}^{p},
$$

i.e. $F$ satisfies (1.2) with $K=m\left\|F^{(m)}\right\|_{\mathscr{L}_{p}}^{p} /[(m-1) !]^{p}$.

We establish the converse implication of Theorem 1.4 in a series of lemmas.

LEMMA 3.1. Let $F$ be a finite-valued function on $(a, b)$, and suppose (1.2) is satisfied for every choice of $n$ and $a<x_{0}<\cdots<x_{n}<b$. Then $F \in C(a, b)$. If $-\infty<a<b<\infty$ then $F$ is bounded on $(a, b)$ and may be extended to a function which is continuous on $[a, b]$.

Proof. Since the lemma follows from Theorem 1.1 for $m=1$, we may assume $m \geqq 2$. Suppose $F$ is discontinuous at the point $x \in(a, b)$. For concreteness, we assume that $F$ is discontinuous from the left, i.e., there exists $\varepsilon>0$ such that $|F(x)-F(y)|>\varepsilon$ for some $y<x$ arbitrarily close to $x$. A discontinuity from the right can be handled similarly. Fix $x=x_{1}<x_{2}<\cdots<x_{m}<b$. For any $a<x_{0}<x_{1}$

$$
\left[F\left(x_{0}\right), \ldots, F\left(x_{m}\right)\right]=\sum_{i=0}^{m}\left[F\left(x_{i}\right) / \prod_{i \neq j=0}^{m}\left(x_{i}-x_{j}\right)\right] .
$$


Thus

$$
\left(x_{1}-x_{0}\right)\left[F\left(x_{0}\right), \ldots, F\left(x_{m}\right)\right]=\frac{-F\left(x_{0}\right)}{\prod_{j=2}^{m}\left(x_{0}-x_{j}\right)}+\frac{F\left(x_{1}\right)}{\prod_{j=2}^{m}\left(x_{1}-x_{j}\right)}+O\left(x_{1}-x_{0}\right)
$$

and hence

$$
\lim _{x_{0} \uparrow x_{1} ;\left|F\left(x_{1}\right)-F\left(x_{0}\right)\right|>\varepsilon}\left(x_{1}-x_{0}\right)\left|\left[F\left(x_{0}\right), \ldots, F\left(x_{m}\right)\right]\right| \geqq \varepsilon /\left|\prod_{j=2}^{m}\left(x_{1}-x_{j}\right)\right| .
$$

This asserts in particular that $x_{0}<x_{1}$ can be chosen such that $\left|\left[F\left(x_{0}\right), \ldots, F\left(x_{m}\right)\right]\right|$ is arbitrarily large. This contradicts the assumption (1.2) for $n=m$ since

$$
\left|\left[F\left(x_{0}\right), \ldots, F\left(x_{m}\right)\right]\right|^{p}\left(x_{m}-x_{0}\right) \geqq\left|\left[F\left(x_{0}\right), \ldots, F\left(x_{m}\right)\right]\right|^{p}\left(x_{m}-x_{1}\right)
$$

and it follows that $F$ is continuous on $(a, b)$. The boundedness of $F$ on $(a, b)$ can be established directly from (1.2) and (3.2) if $(a, b)$ is bounded. Now, to establish the last assertion of the lemma, we will show that $F(a)$ can be defined in such a way that $F$ is continuous from the right at $a$, with a similar argument valid at $b$. Since $F$ is bounded on $(a, b)$ we may choose a sequence $y_{v} \downarrow a$ such that $F\left(y_{v}\right)$ converges, say, $F\left(y_{v}\right) \rightarrow F_{a}$. We define $F(a)=F_{a}$. Now suppose $F$ is not continuous from the right at $a$, i.e., there exist $\varepsilon>0$ and a sequence $z_{v} \downarrow a$ such that $\left|F\left(z_{v}\right)-F(a)\right|>\varepsilon$, and such that $z_{v}<y_{v}$ for all $\nu$. Fix $x_{2}, \ldots, x_{m}$ such that $a<z_{1}<y_{1}<x_{2}<\cdots<x_{m}<b$. By (3.2) we have for all $\nu \geqq 1$,

$$
\left(y_{v}-z_{v}\right)\left[F\left(z_{v}\right), F\left(y_{v}\right), F\left(x_{2}\right), \ldots, F\left(x_{m}\right)\right]=\frac{-F\left(z_{v}\right)}{\prod_{j=\frac{1}{2}}^{m}\left(z_{v}-x_{j}\right)}+\frac{F\left(y_{v}\right)}{\prod_{j=\frac{2}{m}\left(y_{v}-x_{j}\right)}^{m}}+O\left(y_{v}-z_{v}\right) .
$$

Hence,

$$
\lim _{v \rightarrow \infty}\left(y_{v}-z_{v}\right)\left|\left[F\left(z_{v}\right), F\left(y_{v}\right), \ldots, F\left(x_{m}\right)\right]\right| \geqq \varepsilon / \prod_{j=2}^{m}\left(a-x_{j}\right)
$$

As before, this contradicts the assumption (1.2) for $n=m$ and it follows that $\lim F(a+0)=F(a)$. This completes the proof of the lemma.

The remainder of the proof of the converse implication of Theorem 1.4 is subdivided into two cases. We consider first the case when $(a, b)$ is a finite interval.

Lemma 3.2. Let $F \in C[a, b]$ where $-\infty<a<b<\infty$ and let $n \geqq m$. Define $h=$ $(b-a) / n$. There exists $\varphi_{n} \in \mathscr{H}_{p}^{m}$ and a constant $c>0$ independent of $n, a$, and $b$ such that

$$
\begin{gathered}
\varphi_{n}(a+i h)=F(a+i h), \quad 0 \leqq i \leqq n \\
\left\|\varphi_{n}^{(m)}\right\|_{\mathscr{L}_{p}}^{p} \leqq c h \sum_{i=0}^{n-m}\left|\frac{\Delta_{h}^{m} F(a+i h)}{h^{m}}\right|^{p}
\end{gathered}
$$


Proof. It is easily seen by means of a simple affine transformation that we may take $a=0$ and $b=1$. Let $\left\{a_{i}\right\}_{0}^{m-1}$ satisfy the system

$$
\sum_{i=0}^{j} C_{m, j-i}(-1)^{i} \sum_{l=0}^{m-1} a_{l}(i)^{l}=\delta_{j, 0}, \quad 0 \leqq j \leqq m-1,
$$

of $m$ equations, where we have adopted the convention that $0^{0}=1$. This system may be rewritten in the form

$$
A V \mathbf{a}=\boldsymbol{\delta}
$$

where $\mathbf{a}=\left(a_{0}, a_{1}, \ldots, a_{m-1}\right)^{T}$ and $\delta=(1,0,0, \ldots, 0)^{T} . A$ is lower triangular with $A_{j v}=(-1)^{\nu} C_{m, j-v}$ where we take $C_{m, j-v}=0$ if $\nu>j$ and $0 \leqq j \leqq m-1,0 \leqq \nu \leqq m-1$. $V^{\prime}$ is the Vandermonde matrix $V=\left(V_{v^{l}}\right)=\left((\nu)^{l}\right)$ where $0 \leqq \nu \leqq m-1,0 \leqq l \leqq m-1$. Thus (3.6) has a unique solution a since $A V$ is invertible. Define $p(t)=\sum_{i=0}^{m-1} a_{i}(t-m)^{i}$. Let $q(t)=\sum_{i=0}^{3 m-2} b_{i} t^{i}$ be the unique polynomial of degree $3 m-2$ satisfying

Set

$$
\begin{aligned}
q^{(i)}(0) & =0, & & 0 \leqq i \leqq m-1, \\
q^{(i)}(m) & =p^{(i)}(m), & & 0 \leqq i \leqq m-1, \\
q(i) & =0, & & 1 \leqq i \leqq m-1 .
\end{aligned}
$$

$$
\begin{aligned}
G(t) & =p(t), & & m \leqq t<\infty, \\
& =q(t), & & 0 \leqq t<m, \\
& =0, & & t<0,
\end{aligned}
$$

and define $g(t)=G(t / h)$. By the construction of $g$ it is easily seen that for each fixed integer $i$ the function $g_{i}(t)=g(t-i h)$ satisfies

$$
\Delta_{h}^{m} g_{i}(j h)=\delta_{i j} \quad \text { for all integers } j .
$$

Let $\left\{C_{i}\right\}_{0}^{m-1}$ satisfy the system of equations

and define

$$
\sum_{i=0}^{m-1} C_{i}(j h)^{i}=F(j h), \quad 0 \leqq j \leqq m-1,
$$

$$
\varphi_{n}(t)=\sum_{i=0}^{m-1} C_{i} t^{i}+\sum_{i=0}^{n-m}\left\{\Delta_{h}^{m} F(i h)\right\} g_{i}(t) .
$$

$\varphi_{n} \in \mathscr{H}_{p}^{m}(-\infty, \infty)$ and moreover, by (3.7), we have

$$
\Delta_{h}^{m} \varphi_{n}(j h)=\Delta_{h}^{m} F(j h), \quad 0 \leqq j \leqq n-m .
$$

By the choice of the $\left\{C_{i}\right\}_{0}^{m-1} \varphi_{n}$ satisfies $\varphi_{n}(i h)=F(i h), 0 \leqq i \leqq m-1$, and thus by (3.8) it follows that $\varphi_{n}(i h)=F(i h), 0 \leqq i \leqq n$. It remains to verify (3.5).

Clearly,

$$
\varphi_{n}^{(m)}(t)=\sum_{i=\max }^{\min (j, n-m)}\left\{\Delta_{h}^{m} F(i h)\right\} g_{i}^{(m)}(t), \quad j h \leqq t \leqq(j+1) h .
$$


Now

$$
\begin{aligned}
g^{(m)}(t) & =q^{(m)}(t / h) / h^{m}, & & 0 \leqq t \leqq m h, \\
& =0, & & \text { otherwise, }
\end{aligned}
$$

and thus by the Markov-Bernstein theorem

$$
\max _{-\infty<t<\infty}\left|g^{(m)}(t)\right| \leqq K h^{-m},
$$

where $K=(3 m-2)^{2 m} /(m / 2)^{m} \max _{0 \leq t \leq m}|q(t)|$. Hence, for $0 \leqq j \leqq n-1$,

$$
\left|\varphi_{n}^{(m)}(t)\right| \leqq K \sum_{i=\max }^{\min (j, n-m)}\left|\frac{\Delta_{h}^{m} F(i h)}{h^{m}}\right|, \quad j h \leqq t \leqq(j+1) h .
$$

Applying the simple inequality

$$
\left(\sum_{i=1}^{m}\left|\theta_{i}\right|\right)^{p} \leqq\left\{\max _{0 \leqq x \leqq 1} \frac{(1+x)^{p}}{1+x^{p}}\right\}^{m-1} \sum_{i=1}^{m}\left|\theta_{i}\right|^{p}
$$

to the above yields

$$
\left|\varphi_{n}^{(m)}(t)\right|^{p} \leqq K_{1} \sum_{i=\max (0, j-m+1)}^{\min (j, n-m)}\left|\frac{\Delta_{h}^{m} F(i h)}{h^{m}}\right|^{p}, \quad j h \leqq t \leqq(j+1) h,
$$

where

$$
K_{1}=K^{p}\left\{\max _{0 \leqq x \leqq 1} \frac{(1+x)^{p}}{1+x^{p}}\right\}^{m-1}
$$

Finally,

$$
\int_{a}^{b}\left|\varphi_{n}^{(m)}(t)\right|^{p} d t=\sum_{j=0}^{n-1} \int_{j h}^{(j+1) h}\left|\varphi_{n}^{(m)}(t)\right|^{p} d t \leqq m K_{1} h \sum_{\nu=0}^{n-m}\left|\frac{\Delta_{h}^{m} F(\nu h)}{h^{m}}\right|^{p} .
$$

This completes the proof of Lemma 3.2.

We now prove a lemma which extends Theorem 1.2 to $1<p<\infty$ :

Lemma 3.3. Let $F \in C[a, b]$ with $-\infty<a<b<\infty$. Then $F \in \mathscr{H}_{p}^{m}(a, b)$ if and only if there is a constant $K$ independent of $n$ such that on dividing $[a, b]$ into $n$ equal parts by means of the points $x_{i}=a+(b-a) i / n$ and setting $h=(b-a) / n$ we have

$$
h \sum_{i=0}^{n-m}\left|\frac{\Delta_{h}^{m} F\left(x_{i}\right)}{h^{m}}\right|^{p} \leqq K
$$

for all $n$.

Proof. The fact that (3.9) is implied by $F \in \mathscr{H}_{p}^{m}(a, b)$ follows easily from the proof of the first implication of Theorem 1.4. Conversely, suppose (3.9) holds. Then by Lemma 3.2 there exists, for $n \geqq m, \varphi_{n} \in \mathscr{H}_{p}^{m}(a, b)$ satisfying (3.4) and (3.5). Consider the sequence of meshes $\Gamma_{i}=\left\{a+j\left((b-a) / 2^{i}\right)\right\}_{j^{i}=0}^{2^{i}}$ where $2^{i} \geqq m$ and let $s$ 
be the $p$-splines corresponding to the $U_{i}$ of Theorem 2.2. Clearly $\Gamma_{i+1} \supset \Gamma_{i}$ and $\bigcup_{i} \Gamma_{i}$ is dense in $[a, b]$. Moreover, by Lemma 3.2,

$$
\left\|S_{i}^{(m)}\right\|_{\mathscr{L}_{p}}^{p_{p}} \leqq\left\|\varphi_{2^{i}}^{(m)}\right\|_{\mathscr{L}_{p}}^{p} \leqq c h \sum_{j=0}^{2^{i}-m}\left|\frac{\Delta_{h}^{m} F(a+j h)}{h^{m}}\right|^{p} \leqq c K .
$$

Thus, by Theorem $2.2, F \in \mathscr{H}_{p}^{m}$.

We remark for later reference that if (3.9) is satisfied for all $n$ then

$$
\left\|F^{(m)}\right\|_{\mathscr{L}_{p}}^{p} \leqq c K
$$

where $c$ is the constant of Lemma 3.2 and is independent of $n, a$, and $b$.

The converse implication of Theorem 1.4 for a finite interval $(a, b)$ now follows immediately from Lemmas 3.1 and 3.3. Indeed, if (1.2) holds then by Lemma 3.1 $F \in C[a, b]$. In this case (1.2) holds for every choice of $n$ and $a \leqq x_{0}<x_{1}<\cdots$ $<x_{n} \leqq b$ and thus the hypotheses of Lemma 3.3 are satisfied, so that $F \in \mathscr{H}_{p}^{m}(a, b)$.

We now consider the case where $(a, b)$ is an infinite interval. Suppose then that (1.2) holds. In particular it holds for any fixed finite interval $(\alpha, \beta)$. By the above, $F \in \mathscr{H}_{p}^{m}(\alpha, \beta)$ with $\left\|F^{(m)}\right\|_{\mathscr{L}_{p}}^{p} \leqq c K$ where $c K$ is independent of $(\alpha, \beta)$. By Fatou's Lemma we conclude that $\int_{a}^{b}\left|F^{(m)}(x)\right|^{p} d x \leqq c K$, i.e., $F \in \mathscr{H}_{p}^{m}(a, b)$. This completes the proof of Theorem 1.4.

Arguing analogously from Lemma 3.3 we can immediately deduce

Theorem 3.4. Let $F \in C(-\infty, \infty)$. Then $F \in \mathscr{H}_{p}^{m}(-\infty, \infty)$ if and only if there is a constant $K$ independent of $h$ such that

$$
h \sum_{-\infty}^{\infty}\left|\frac{\Delta_{h}^{m} F(i h)}{h^{m}}\right|^{p} \leqq K
$$

for all $h>0$.

A similar result holds for semi-infinite intervals.

We conclude the paper with a summary of some of the results. These are contained in

THEOREM 3.5. Let $(a, b)$ be a possibly infinite interval and let $F$ be a finite-valued function on $(a, b)$. Then the following three statements are equivalent.

(a) $F \in \mathscr{H}_{p}^{m}(a, b)$;

(b) F satisfies (1.2) for every choice of $n$ and $a<x_{0}<\cdots<x_{n}<b$;

(c) there exists a constant $K>0$ such that for every choice of $n$ and $a<x_{0}<\cdots$ $<x_{n}<b$ the $p$-splines $s$ interpolating the values $F\left(x_{0}\right), \ldots, F\left(x_{n}\right)$ satisfy $\left\|s^{(m)}\right\|_{\mathscr{L}_{p}} \leqq K$.

Proof. We have shown that (a) $\Leftrightarrow$ (b) and (a) $\Rightarrow$ (c). We now show that (c) $\Rightarrow$ (b). Notice that

$$
\sum_{i=0}^{n-m}\left|\left[F\left(x_{i}\right), \ldots, F\left(x_{i+m}\right)\right]\right|^{p}\left(x_{i+m}-x_{i}\right) \equiv \sum_{i=0}^{n-m}\left|\left[s\left(x_{i}\right), \ldots, s\left(x_{i+m}\right)\right]\right|^{p}\left(x_{i+m}-x_{i}\right)
$$


where $s$ is the interpolating $p$-spline function of (c). But $s \in \mathscr{H}_{p}^{m}(a, b)$ and by Theorem 1.4

$$
\sum_{i=0}^{n-m}\left|\left[s\left(x_{i}\right), \ldots, s\left(x_{i+m}\right)\right]\right|^{p}\left(x_{i+m}-x_{i}\right) \leqq \frac{m}{[(m-1) !]^{p}}\left\|s^{(m)}\right\|_{\mathscr{L}_{p}}^{p} \leqq m\left[\frac{K}{(m-1) !}\right]^{p} .
$$

ACKnowledgement. We are indebted to Professor I. J. Schoenberg for bringing this problem to our attention. Recently, Professor S. Karlin has discovered an alternative proof of Theorem 1.4 which obtains the result directly without utilizing the properties of $p$-splines.

\section{REFERENCES}

1. S. Goldberg, Unbounded linear operators, McGraw-Hill, New York, 1966.

2. J. Jerome and L. Schumaker, Lg-splines, J. Approximation Theory 2 (1969), $29-49$.

3. F. Riesz, Systeme integrierbarer Funktionen, Math. Ann. 69 (1910), 449-497.

4. F. Riesz and B. Sz.-Nagy, Functional analysis, Ungar, New York, 1955.

5. I. J. Schoenberg, Spline interpolation and the higher derivatives, Proc. Nat. Acad. Sci. U.S.A. 51 (1964), 24-28.

Mathematics Research Center, United States Army, University of Wisconsin, MADISON, Wisconsin 